Research Paper

\title{
Marital Status and Survival in Patients with Penile Cancer
}

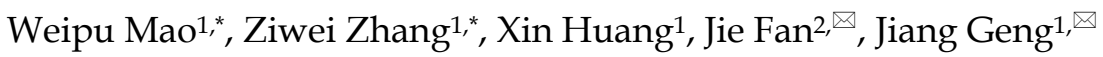 \\ 1. Department of Urology, Shanghai Tenth People's Hospital, Tongii University, Shanghai, 200072, China \\ 2. Department of Pathology, Huashan Hospital, Fudan University, Shanghai, 200040, China \\ ${ }^{*}$ First author \\ $\triangle$ Corresponding authors: Professor Jiang Geng, Shanghai Tenth People's Hospital, Tongji University, 301 Middle Yanchang Road, Shanghai, 200072, China. \\ Tel: 86-021-66307508; Fax: 86-021-66307508; E-mail: gengjiangsn@sina.com and Jie Fan, Department of Pathology, Huashan Hospital, Fudan University, \\ Shanghai, 200040, China, E-mail: fanfanj369@sina.com \\ (1) Ivyspring International Publisher. This is an open access article distributed under the terms of the Creative Commons Attribution (CC BY-NC) license \\ (https://creativecommons.org/licenses/by-nc/4.0/). See http://ivyspring.com/terms for full terms and conditions.
}

Received: 2018.12.05; Accepted: 2019.04.27; Published: 2019.06.02

\begin{abstract}
Purpose: We aimed to reveal the effects of marital status on survival outcomes in patients with penile cancer.

Methods: Patients with penile cancer who were diagnosed between 2004 and 2015 were identified by using the Surveillance, Epidemiology and End Results (SEER) database. Kaplan-Meier and Cox regressions were used to analyse the effects of marital status on overall survival (OS) and cancer-specific survival (CSS).

Results: Among 3,195 eligible patients with penile cancer, 1,951 (61.1\%) patients were married, 365 (11.4\%) were divorced or separated, $327(10.2 \%)$ were widowed and $552(17.3 \%)$ were single. The widowed patients had the worst OS median survival time (22 months) and CSS median survival time (23.5 months). Marital status was an independent prognostic factor for OS and CSS of penile cancer patients. The multivariate Cox regression showed that widowed patients exhibited the poorest OS (hazard ratio [HR]: 1.73; 95\% confidence interval $[\mathrm{Cl}]: 1.48-2.03, p<0.001$ ) and the poorest CSS (HR: $1.64 ; 95 \% \mathrm{Cl}: 1.144-1.279, p<0.001$ ) compared with married patients. Similar results were observed in our centre database and the subgroup analyses based on the SEER stage and grade.

Conclusions: In our study, we found that marital status was an independent prognostic factor for survival in patients with penile cancer. Additionally, widowed patients had the lowest OS and CSS compared with married patients.
\end{abstract}

Key words: penile cancer; marital status; cancer survival; SEER

\section{Introduction}

Penile cancer is a rare malignant tumour in the genitourinary system and mostly occurs in middle-aged men aged 50-70 years [1]. The incidence of penile cancer varies from region to region, due to different countries, nationalities, religions and hygienic habits [2]. Penile cancer accounts for $0.4 \%-0.6 \%$ of all male malignancies in Europe and the United States, but it accounts for as much as $10 \%$ of malignancies in economically underdeveloped parts of Asia, Africa and South America [3-5].

Phimosis and prepuce are the more recognized risk factors for penile cancer, which then leads to the prepuce and penile head in the chronic stimulation environment, with a long-term, local inflammatory response [6]. Multiple sexual partners, genital warts or other sexually transmitted diseases are also risk factors for penile cancer [3].

Previous studies have shown that marital status is an independent prognostic factor for the survival of multiple cancers, including colorectal cancer [7], breast cancer [8], stomach cancer [9] and pancreatic cancer [10]. In this study, our purpose was to investigate the impact of marital status on the survival of penile cancer patients, based on a large amount of population data that was gathered using the 
Surveillance, Epidemiology, and End Results (SEER) database.

\section{Patients and Methods}

\section{Data source and patients}

Via the National Cancer Institute's SEER*Stat software (version 8.3.5; SEER 18 Regs Custom Data [with an additional treatment field], November 2017 Sub [1973-2015 varying] database), we identified 3,983 penile cancer patients with known marital statuses between January 1, 2004, and December 31, 2015. The SEER database covers approximately $28 \%$ of the US population and collects information on cancer patients, including demographic information, primary tumour locations, cancer stages, treatment modalities and survival times [11].

The exclusion criteria in our study were as follows: (a) an unknown marital status; (b) an unknown survival time; (c) an unknown AJCC stage; (d) an unknown T/N/M stage; (e) patients who were under 18 years of age and (f) patients who were diagnosed at autopsy. Finally, we identified 3,195 eligible patients who were diagnosed with penile cancer.

A total of 69 patients had diagnoses of penile cancer at the Shanghai Tenth People's Hospital of Tongji University (Shanghai, China) between January 1, 2013, and December 31, 2017. Patients received follow-ups until December 31, 2018. This study was approved by the Ethics Committee of Shanghai Tenth People's Hospital of Tongii University, and written informed consent was obtained from all the patients or their relatives.

\section{Study variables}

The study variables in our study included the age of diagnosis, race, urban-rural residence, tumour primary site, tumour grade, histologic type, SEER stage, AJCC stage, TNM stage and surgical information. According to the marital status, we divided patients into four groups: married, divorced or separated, widowed, and single. The clinicopathological characteristics included age at diagnosis ( $\leq 60$ and $>60$ ), race (white, black, and other) and urban-rural residence (metropolitan and nonmetropolitan). The tumour variables included the tumour primary site (prepuce, glans penis, body of penis, overlapping lesion, and penis NOS), histological type (squamous cell carcinoma, verrucous carcinoma, and others), SEER stage (localized, regional, distant, and unknown), AJCC stage (0, I, II, III, and IV), T-stage (Ta, T1, T2, T3, and T4), N-stage (N0, N1, N2, and N3), M-stage (M0 and M1), and treatment (surgery and no surgery). Tumour grades I-IV represented well differentiated, moderately differentiated, poorly differentiated, and undifferentiated tumours, respectively. Overall survival (OS) and cancer-specific survival (CSS) were the primary study end points in this study.

\section{Statistical analysis}

The chi-square test was used to analyse the factors related to marital status. Kaplan-Meier analyses, with log-rank tests, were used to analyse the OS and CSS rates of patients with different marital statuses and other variables. A multivariable Cox regression was used to determine the factors associated with OS and CSS. Statistical Package for the Social Sciences software (version 20.0; SPSS Inc, Chicago, IL, USA) was used for all the statistical analyses. A $p$ value $\leq 0.05$ was considered to be statistically significant.

\section{Results}

\section{Demographic and clinical characteristics of the penile cancer}

A total of 3,195 eligible penile cancer patients were included in our study cohort through the SEER database from 2004 to 2015. Among them, 1,951 $(61.1 \%)$ patients were married, $365(11.4 \%)$ were divorced or separated, $327(10.2 \%)$ were widowed, and $552(17.3 \%)$ were single. Table 1 shows the clinical characteristics and the chi-square test results for the comparison of penile cancer with different marital statuses. The Chi-square test exhibited significant differences between the different marital statuses in several variables, including the age at diagnosis $(p<0.001)$, race $(p<0.001)$, tumour primary site $(p=0.014)$, tumour grade $(p=0.023)$, T-stage $(p=$ $0.031)$, N-stage $(p=0.044)$ and surgical treatment $(p=$ 0.011). White patients accounted for the majorities of each of the groups, but the proportion of black patients was higher in the single group than in the other groups. The widowed patients were more likely to be over 60 years $(95.4 \%)$, while most of the single patients were under 60 years $(51.3 \%)$. In addition, the primary site of penile cancer in the widowed patients is less common in the body of the penis.

\section{Effects of different variables on OS and CSS in patients with penile cancer}

In our study, Kaplan-Meier curves were used to analyse the influences of related factors on the OS and CSS of patients with penile cancer (Table 2). The median OS time of the married group was 33 months, while the median OS times of the divorced/separated, widowed and single groups were 28 months, 22 months and 26 months, respectively. The survival times of the four marital subgroups were significantly different $(p<0.001)$ (Fig. 1a). In addition, the median 
CSS time of the married group was 38 months, while the median OS times of the divorced/separated, widowed and single groups were 34 months, 23.5 months and 29 months, respectively $(p<0.001)$ (Fig. 1b). The widowed patients had the worst OS and CSS.
In addition, the Kaplan-Meier analysis showed that the age at diagnosis, histological type, SEER stage, tumour grade, AJCC stage, TNM stage and surgical therapy were also significantly correlated with OS and CSS.

Table 1. Characteristics for penile cancer patients stratified by marital status.

\begin{tabular}{|c|c|c|c|c|c|c|}
\hline \multirow[t]{2}{*}{ Characteristic } & \multirow{2}{*}{$\begin{array}{l}\text { Total } \\
\text { No. }(\%)\end{array}$} & \multirow{2}{*}{$\begin{array}{l}\text { Married } \\
\text { No. }(\%)\end{array}$} & \multicolumn{2}{|c|}{ Divorced/Separated Widowed } & \multirow{2}{*}{$\begin{array}{l}\text { Single } \\
\text { No. }(\%)\end{array}$} & \multirow[t]{2}{*}{ P value } \\
\hline & & & No. $(\%)$ & No. $(\%)$ & & \\
\hline Total & 3195 & 1951 (61.1) & $365(11.4)$ & $327(10.2)$ & $552(17.3)$ & \\
\hline Age at diagnosis & & & & & & $<0.001$ \\
\hline$<60$ & $938(29.4)$ & $534(27.4)$ & $106(29.0)$ & $15(4.6)$ & $283(51.3)$ & \\
\hline$\geq 60$ & $2257(70.6)$ & 1417 (72.6) & $259(71.0)$ & $312(95.4)$ & $269(48.7)$ & \\
\hline Race & & & & & & $<0.001$ \\
\hline White & $2678(83.8)$ & $1675(85.9)$ & $311(85.2)$ & $279(85.3)$ & $413(74.8)$ & \\
\hline Black & $328(10.3)$ & $144(7.4)$ & 47 (12.9) & $34(10.4)$ & $103(18.7)$ & \\
\hline Other & $189(5.9)$ & $132(6.8)$ & $7(1.9)$ & $14(4.3)$ & $36(6.5)$ & \\
\hline Urban-rural residence & & & & & & 0.056 \\
\hline Metropolitan & $2654(83.1)$ & $1626(83.3)$ & $286(78.4)$ & $280(85.6)$ & $462(83.7)$ & \\
\hline Nonmetropolitan & $541(16.9)$ & $325(16.7)$ & $79(21.6)$ & $47(14.4)$ & $90(16.3)$ & \\
\hline Primary site & & & & & & 0.014 \\
\hline Prepuce & $382(12.0)$ & $253(13.0)$ & $39(10.7)$ & 38 (11.6) & $52(9.4)$ & \\
\hline Glans penis & 1070 (33.5) & $684(35.1)$ & $107(29.3)$ & $107(32.7)$ & $172(31.2)$ & \\
\hline Body of penis & $175(5.5)$ & $103(5.3)$ & $29(7.9)$ & $10(3.1)$ & $33(6.0)$ & \\
\hline Overlapping lesion & $139(4.4)$ & $85(4.4)$ & $13(3.6)$ & $13(4.0)$ & $28(5.1)$ & \\
\hline Penis, NOS & 1429 (44.7) & $826(42.3)$ & $177(48.5)$ & $159(48.6)$ & $267(48.4)$ & \\
\hline Histological Type & & & & & & 0.071 \\
\hline Squamous cell carcinoma & $2879(90.1)$ & $1738(89.1)$ & $332(91.0)$ & $299(91.4)$ & $510(92.4)$ & \\
\hline Verrucous carcinoma & $173(5.4)$ & $116(5.9)$ & $17(4.7)$ & $11(3.4)$ & $29(5.3)$ & \\
\hline Others & $143(4.5)$ & $97(5.0)$ & $16(4.4)$ & $17(5.2)$ & $13(2.4)$ & \\
\hline SEER stage & & & & & & 0.248 \\
\hline Localized & 1978 (61.9) & $1233(63.2)$ & $218(59.7)$ & $210(64.2)$ & $317(57.4)$ & \\
\hline Regional & $1028(32.2)$ & $606(31.1)$ & $123(33.7)$ & $102(31.2)$ & $197(35.7)$ & \\
\hline Distant & $187(5.9)$ & $111(5.7)$ & $23(6.3)$ & $15(4.6)$ & $38(6.9)$ & \\
\hline Unstaged & $2(0.1)$ & $1(0.1)$ & $1(0.3)$ & $0(0.0)$ & $0(0.0)$ & \\
\hline Grade & & & & & & 0.023 \\
\hline Grade I/Grade II & $2030(63.5)$ & $1240(63.6)$ & $212(58.1)$ & $202(61.8)$ & $376(68.1)$ & \\
\hline Grade III/Grade IV & $612(19.2)$ & $359(18.4)$ & $83(22.7)$ & $65(19.9)$ & $105(19.0)$ & \\
\hline Unknown & $553(17.3)$ & $352(18.0)$ & $70(19.2)$ & $60(18.3)$ & $71(12.9)$ & \\
\hline AJCC stage & & & & & & 0.123 \\
\hline 0 & $25(0.8)$ & $16(0.8)$ & $2(0.5)$ & $2(0.6)$ & $5(0.9)$ & \\
\hline I & 1708 (53.5) & $1064(54.5)$ & $194(53.2)$ & $179(54.7)$ & $271(49.1)$ & \\
\hline II & $661(20.7)$ & $407(20.9)$ & $65(17.8)$ & 75 (22.9) & $114(20.7)$ & \\
\hline III & $519(16.2)$ & $311(15.9)$ & 69 (18.9) & $42(12.8)$ & 97 (17.6) & \\
\hline IV & $282(8.8)$ & $153(7.8)$ & $35(9.6)$ & $29(8.9)$ & 65 (11.8) & \\
\hline T-stage & & & & & & 0.031 \\
\hline $\mathrm{Ta}$ & $25(0.8)$ & $16(0.8)$ & $2(0.5)$ & $2(0.6)$ & $5(0.9)$ & \\
\hline $\mathrm{T} 1$ & 1869 (58.5) & $1162(59.6)$ & $213(58.4)$ & $190(58.1)$ & $304(55.1)$ & \\
\hline $\mathrm{T} 2$ & $728(22.8)$ & $451(23.1)$ & $74(20.3)$ & $80(24.5)$ & $123(22.3)$ & \\
\hline $\mathrm{T} 3$ & $487(15.2)$ & $281(14.4)$ & 69 (18.9) & $42(12.8)$ & 95 (17.2) & \\
\hline $\mathrm{T} 4$ & $86(2.7)$ & $41(2.1)$ & $7(1.9)$ & $13(4.0)$ & $25(4.5)$ & \\
\hline $\mathrm{N}$-stage & & & & & & 0.034 \\
\hline N0 & $2620(82.0)$ & 1619 (83.0) & $285(78.1)$ & $278(85.0)$ & $438(79.3)$ & \\
\hline N1 & $193(6.0)$ & $104(5.3)$ & $31(8.5)$ & $22(6.7)$ & $36(6.5)$ & \\
\hline N2 & $214(6.7)$ & $129(6.6)$ & $31(8.5)$ & $10(3.1)$ & $44(8.0)$ & \\
\hline N3 & $168(5.3)$ & $99(5.1)$ & $18(4.9)$ & $17(5.2)$ & $34(6.2)$ & \\
\hline M-stage & & & & & & 0.093 \\
\hline M0 & 3097 (96.9) & 1902 (97.5) & 350 (95.9) & 317 (96.9) & $528(95.7)$ & \\
\hline M1 & $98(3.1)$ & $49(2.5)$ & $15(4.1)$ & $10(3.1)$ & $24(4.3)$ & \\
\hline Surgical therapy & & & & & & 0.011 \\
\hline No & $200(6.3)$ & $101(5.2)$ & $33(9.0)$ & $25(7.6)$ & $41(7.4)$ & \\
\hline Yes & 1995 (93.7) & $1850(94.8)$ & $332(91.0)$ & $302(92.4)$ & $511(92.6)$ & \\
\hline
\end{tabular}


a.

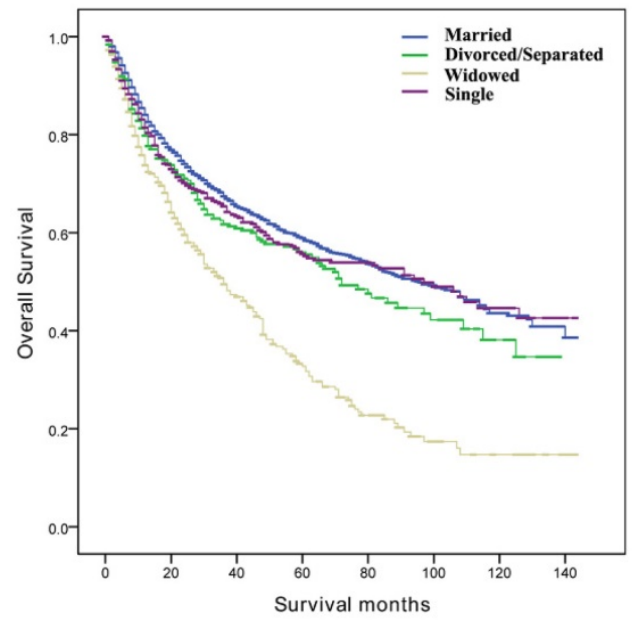

b.

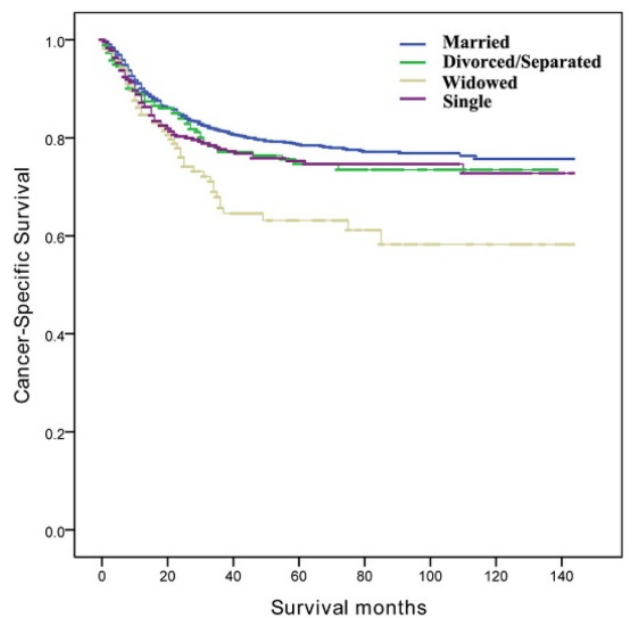

Figure 1. Kaplan-Meier survival curves according to marital status (married, divorced/separated, widowed, and single) in patients with penile cancer. a, Overall survival; b, cancer-specific survival.

Table 2. Kaplan-Meier analysis overall survival and cancer-specific survival for penile cancer patients.

\begin{tabular}{|c|c|c|c|c|c|c|}
\hline \multirow[t]{2}{*}{ Characteristic } & \multirow{2}{*}{$\begin{array}{l}\text { OS MST } \\
\text { (months) }\end{array}$} & \multicolumn{2}{|c|}{ Kaplan-Meier } & \multirow{2}{*}{$\begin{array}{l}\text { CSS MST } \\
\text { (months) }\end{array}$} & \multicolumn{2}{|c|}{ Kaplan-Meier } \\
\hline & & $\begin{array}{l}\text { Log } \\
\text { Rank } \chi^{2} \\
\text { test }\end{array}$ & $\begin{array}{l}P \\
\text { value }\end{array}$ & & $\begin{array}{l}\log \\
\text { Rank } \chi^{2} \\
\text { test }\end{array}$ & $\begin{array}{l}P \\
\text { value }\end{array}$ \\
\hline Age at diagnosis & & 114.547 & $<0.001$ & & 18.655 & $<0.001$ \\
\hline$<60$ & 38 & & & 42 & & \\
\hline$\geq 60$ & 27 & & & 31 & & \\
\hline Marital status & & 82.663 & $<0.001$ & & 14.738 & 0.002 \\
\hline Married & 33 & & & 38 & & \\
\hline Divorced/Separated & 28 & & & 34 & & \\
\hline Widowed & 22 & & & 23.5 & & \\
\hline Single & 26 & & & 29 & & \\
\hline Histological Type & & 30.477 & $<0.001$ & & 32.174 & $<0.001$ \\
\hline $\begin{array}{l}\text { Squamous cell } \\
\text { carcinoma }\end{array}$ & 28 & & & 33 & & \\
\hline Verrucous carcinoma & 48 & & & 54 & & \\
\hline Others & 35 & & & 49 & & \\
\hline SEER stage & & 347.495 & $<0.001$ & & 468.526 & $<0.001$ \\
\hline Localized & 38 & & & 44 & & \\
\hline Regional & 23 & & & 25 & & \\
\hline Distant & 8 & & & 9 & & \\
\hline Grade & & 83.892 & $<0.001$ & & 84.244 & $<0.001$ \\
\hline Grade I/Grade II & 31 & & & 36 & & \\
\hline Grade III/Grade IV & 20 & & & 20 & & \\
\hline Unknown & 42 & & & 48 & & \\
\hline \multicolumn{7}{|l|}{ AJCC stage } \\
\hline 0 & 42 & 293.283 & $<0.001$ & & 398.246 & $<0.001$ \\
\hline I & 39 & & & 48.5 & & \\
\hline II & 29 & & & 45 & & \\
\hline III & 22 & & & 31 & & \\
\hline IV & 9.5 & & & 24.5 & & \\
\hline T-stage & & 111.027 & $<0.001$ & & 158.953 & $<0.001$ \\
\hline $\mathrm{Ta}$ & 42 & & & 48.5 & & \\
\hline $\mathrm{T} 1$ & 36 & & & 42 & & \\
\hline $\mathrm{T} 2$ & 26 & & & 28 & & \\
\hline T3 & 20 & & & 23 & & \\
\hline $\mathrm{T} 4$ & 13 & & & 17.5 & & \\
\hline $\mathrm{N}$-stage & & 261.080 & $<0.001$ & & 438.318 & $<0.001$ \\
\hline No & 35 & & & 41 & & \\
\hline N1 & 17 & & & 18 & & \\
\hline N2 & 14.5 & & & 14.5 & & \\
\hline N3 & 10 & & & 10.5 & & \\
\hline M-stage & & 205.009 & $<0.001$ & & 221.247 & $<0.001$ \\
\hline M0 & 31 & & & 36 & & \\
\hline M1 & 7 & & & 8 & & \\
\hline
\end{tabular}

\begin{tabular}{|c|c|c|c|c|c|c|}
\hline \multirow[t]{2}{*}{ Characteristic } & \multirow{2}{*}{$\begin{array}{l}\text { OS MST } \\
\text { (months) }\end{array}$} & \multicolumn{2}{|c|}{ Kaplan-Meier } & \multirow{2}{*}{$\begin{array}{l}\text { CSS MST } \\
\text { (months) }\end{array}$} & \multicolumn{2}{|c|}{ Kaplan-Meier } \\
\hline & & $\begin{array}{l}\text { Log } \\
\text { Rank } X^{2} \\
\text { test }\end{array}$ & $\begin{array}{l}P \\
\text { value }\end{array}$ & & $\begin{array}{l}\text { Log } \\
\text { Rank } X^{2} \\
\text { test }\end{array}$ & $\begin{array}{l}P \\
\text { value }\end{array}$ \\
\hline Surgical therapy & & 77.741 & $<0.001$ & & 42.611 & $<0.001$ \\
\hline No & \multicolumn{3}{|l|}{13} & \multicolumn{3}{|l|}{16} \\
\hline Yes & \multicolumn{3}{|l|}{31} & \multicolumn{3}{|l|}{36} \\
\hline \multicolumn{7}{|c|}{$\begin{array}{l}\text { Abbreviations: MST, median survival time; OS, overall survival; CSS, cause-specific } \\
\text { survival; SEER, Surveillance, Epidemiology and End Results; Grade I, Well } \\
\text { differentiated; Grade II, Moderately differentiated; Grade III, Poorly differentiated; } \\
\text { Grade IV, Undifferentiated; AJCC, American Joint Committee on Cancer }\end{array}$} \\
\hline
\end{tabular}

Univariate and multivariate cox regressions were used to analyse the factors associated with OS and CSS. As shown in Table 3, the age at diagnosis, marital status, histological type, tumour grade, SEER stage, $\mathrm{N}$ stage, $\mathrm{M}$ stage, and surgical therapy were factors that affected OS and CSS (all $p<0.05$ ). According to the multivariate Cox regression, divorced/separated subjects (vs married; $H R=1.11$, 95\% CI: 0.93-1.32, $p=0.238$ ), widowed subjects (vs married; HR=1.73, 95\% CI: 1.48-2.03, $p<0.001)$ and single subjects (vs married; $H R=1.20,95 \% \mathrm{CI}$ : $1.02-1.40, p=0.026$ ) were associated with significantly greater odds of OS. In terms of CSS, the multivariate Cox regression analysis also indicated that marriage was a significant protective factor for penile cancer patients (separated/divorced: HR, 0.95; 95\% CI, 0.70-1.28; $p=0.743$; widowed: HR, $1.64 ; 95 \% \mathrm{CI}$, 1.19-2.25; $p=0.002$; single: HR, 1.24; 95\% CI, 0.97-1.60; $p=0.092$ ).

\section{Subgroup analysis for evaluating the effect of marital status on OS and CSS based on SEER stage and grade}

Based on the SEER stage and grade, we further discussed the difference between marital status and 
prognosis among the subgroups of the penile cancer patients (Table 4). We observed that, for OS and CSS, marital status was still an independent prognostic factor for localized and Grade I/Grade II subgroups (Fig. 2a, b and Fig. 3a, b). However, for the distant and Grade III/Grade IV subgroup patients, the effect of marital status on OS and CSS was not significant (Fig. 2e, $f$ and Fig. 3c, d).

Table 3. Risk factors for survival: outcome is overall survival and penis cancer-specific survival.

\begin{tabular}{|c|c|c|c|c|}
\hline \multirow[t]{2}{*}{ Characteristic } & \multicolumn{2}{|l|}{ OS } & \multicolumn{2}{|l|}{ CSS } \\
\hline & $\begin{array}{l}\text { Hazard Ratio } \\
(95 \% \mathrm{CI})\end{array}$ & P value & $\begin{array}{l}\text { Hazard Ratio } \\
(95 \% \mathrm{CI})\end{array}$ & P value \\
\hline \multicolumn{5}{|l|}{ Age at diagnosis } \\
\hline$<60$ & Reference & & Reference & \\
\hline$\geq 60$ & $2.04(1.77-2.36)$ & $<0.001$ & $1.69(1.35-2.10)$ & $<0.001$ \\
\hline \multicolumn{5}{|l|}{ Marital status } \\
\hline Married & Reference & & Reference & \\
\hline Divorced/Separated & $1.11(0.93-1.32)$ & 0.238 & $0.95(0.70-1.28)$ & 0.743 \\
\hline Widowed & $1.73(1.48-2.03)$ & $<0.001$ & $1.64(1.19-2.25)$ & 0.002 \\
\hline Single & $1.20(1.02-1.40)$ & 0.026 & $1.24(0.97-1.60)$ & 0.092 \\
\hline \multicolumn{5}{|l|}{ Histological Type } \\
\hline Squamous cell carcinoma & Reference & & Reference & \\
\hline Verrucous carcinoma & $0.59(0.43-0.81)$ & 0.001 & $0.26(0.10-0.66)$ & 0.005 \\
\hline Others & $1.10(0.83-1.45)$ & 0.512 & $0.58(0.27-1.28)$ & 0.179 \\
\hline \multicolumn{5}{|l|}{ SEER stage } \\
\hline Localized & Reference & & Reference & \\
\hline Regional & $1.39(1.21-1.60)$ & $<0.001$ & $1.20(0.79-1.82)$ & 0.467 \\
\hline Distant & $2.46(1.66-3.64)$ & $<0.001$ & $1.98(1.02-3.84)$ & 0.044 \\
\hline \multicolumn{5}{|l|}{ Grade } \\
\hline Grade I/Grade II & Reference & & Reference & \\
\hline Grade III/Grade IV & $1.21(1.06-1.39)$ & 0.005 & $1.32(1.06-1.64)$ & 0.012 \\
\hline Unknown & $0.84(0.71-0.99)$ & 0.042 & $0.81(0.58-1.13)$ & 0.210 \\
\hline \multicolumn{5}{|l|}{ AJCC stage } \\
\hline 0 & Reference & & Reference & \\
\hline I & NA & 0.113 & $0.54(0.07-4.26)$ & 0.555 \\
\hline II & NA & 0.735 & $1.05(0.13-8.50)$ & 0.967 \\
\hline III & NA & 0.632 & $1.14(0.14-9.47)$ & 0.907 \\
\hline IV & NA & 0.227 & $1.30(0.15-11.27)$ & 0.812 \\
\hline \multicolumn{5}{|l|}{ T-stage } \\
\hline $\mathrm{Ta}$ & Reference & & Reference & \\
\hline $\mathrm{T} 1$ & NA & 0.095 & NA & 0.360 \\
\hline $\mathrm{T} 2$ & NA & 0.796 & NA & 0.773 \\
\hline $\mathrm{T} 3$ & NA & 0.294 & NA & 0.718 \\
\hline $\mathrm{T} 4$ & NA & 0.027 & NA & 0.069 \\
\hline \multicolumn{5}{|l|}{$\mathrm{N}$-stage } \\
\hline No & Reference & & Reference & \\
\hline N1 & 1.35 (1.08-1.69) & 0.008 & $2.10(1.49-2.97)$ & $<0.001$ \\
\hline N2 & $1.54(1.24-1.92)$ & $<0.001$ & $2.23(1.59-3.140$ & $<0.001$ \\
\hline N3 & $1.65(1.21-2.24)$ & 0.001 & $2.17(1.32-3.58)$ & 0.002 \\
\hline \multicolumn{5}{|l|}{ M-stage } \\
\hline M0 & Reference & & Reference & \\
\hline M1 & $1.56(1.07-2.29)$ & 0.021 & $1.94(1.18-3.19)$ & 0.009 \\
\hline \multicolumn{5}{|l|}{ Surgical therapy } \\
\hline No & Reference & & Reference & \\
\hline Yes & $0.56(0.46-0.68)$ & $<0.001$ & $0.52(0.38-0.72)$ & $<0.001$ \\
\hline
\end{tabular}

\section{Discussion}

Our study explored the effect of marital status on OS and CSS in penile cancer patients and found that marital status was an independent prognostic factor for the prognosis of penile cancer. Marital status has a protective effect on the survival outcome of penile cancer, which was consistent with previous studies on other types of cancer [12-14].

Table 4. Median survival months and survival months of penile cancer patients.

\begin{tabular}{|c|c|c|c|c|c|c|}
\hline \multirow[t]{2}{*}{ Characteristic } & \multirow{2}{*}{$\begin{array}{l}\text { OS MST } \\
\text { (months) }\end{array}$} & \multicolumn{2}{|c|}{ Kaplan-Meier } & \multirow{2}{*}{$\begin{array}{l}\text { CSS MST } \\
\text { (months) }\end{array}$} & \multicolumn{2}{|c|}{ Kaplan-Meier } \\
\hline & & $\begin{array}{l}\text { Log Rank } \\
\chi^{2} \text { test }\end{array}$ & $\begin{array}{l}\mathbf{P} \\
\text { value }\end{array}$ & & $\begin{array}{l}\text { Log Rank } \\
X^{2} \text { test }\end{array}$ & $\begin{array}{l}P \\
\text { value }\end{array}$ \\
\hline Localized & & 95.335 & $<0.001$ & & 12.127 & 0.007 \\
\hline Married & 40.0 & & & 54.0 & & \\
\hline Divorced/Separated & 40.0 & & & 49.5 & & \\
\hline Widowed & 25.0 & & & 36.0 & & \\
\hline Single & 39.0 & & & 45.0 & & \\
\hline Regional & & 11.060 & 0.011 & & 5.024 & 0.170 \\
\hline Married & 25.0 & & & 42.0 & & \\
\hline Divorced/Separated & 19.0 & & & 30.5 & & \\
\hline Widowed & 21.0 & & & 32.0 & & \\
\hline Single & 20.0 & & & 32.0 & & \\
\hline Distant & & 4.864 & 0.182 & & 2.212 & 0.530 \\
\hline Married & 9.0 & & & 19.0 & & \\
\hline Divorced/Separated & 8.0 & & & 13.5 & & \\
\hline Widowed & 5.0 & & & 5.0 & & \\
\hline Single & 8.5 & & & 25.0 & & \\
\hline Grade I/Grade II & & 54.129 & $<0.001$ & & 12.052 & 0.007 \\
\hline Married & 35.0 & & & 49.0 & & \\
\hline Divorced/Separated & 28.0 & & & 43.0 & & \\
\hline Widowed & 23.0 & & & 31.0 & & \\
\hline Single & 28.0 & & & 39.0 & & \\
\hline Grade III/Grade IV & & 4.431 & 0.219 & & 5.499 & 0.139 \\
\hline Married & 20.0 & & & 32.0 & & \\
\hline Divorced/Separated & 19.0 & & & 37.0 & & \\
\hline Widowed & 20.0 & & & 31.0 & & \\
\hline Single & 15.0 & & & 33.0 & & \\
\hline
\end{tabular}

Our study showed that the OS and CSS times of the married patients were significantly better than the unmarried patients, including the divorced/ separated, the widowed and the single patients. In addition, the widowed patients had the lowest OS and CSS times (22 months and 23.5 months, respectively) compared with the other groups. After adjusting for the age at diagnosis, histological type, tumour grade, SEER stage, AJCC stage, TNM stage and surgical therapy, the multivariable Cox regression analysis showed that married patients had the highest OS and CSS times, and the widowed patients had the worst OS and CSS times. The stratified analysis showed that the married patients had better survival outcomes of the localized and Grade I/Grade II subgroup patients, while the widowed patients had worse survival outcomes.

We suspect that the fact that the widowed patients with the worst OS and CSS times may be related to the following factors. First, married patients are in better health than other groups, which may be related to their better family and higher incomes [15, 16]. Second, widowed patients may have more complicated mental and psychological factors, and 
mental pressure is more likely to cause depression [17, 18]. In addition, widowed patients may receive less care after being diagnosed with cancer, thus increasing their mortality [19, 20]. Finally, widowed

\section{a.}

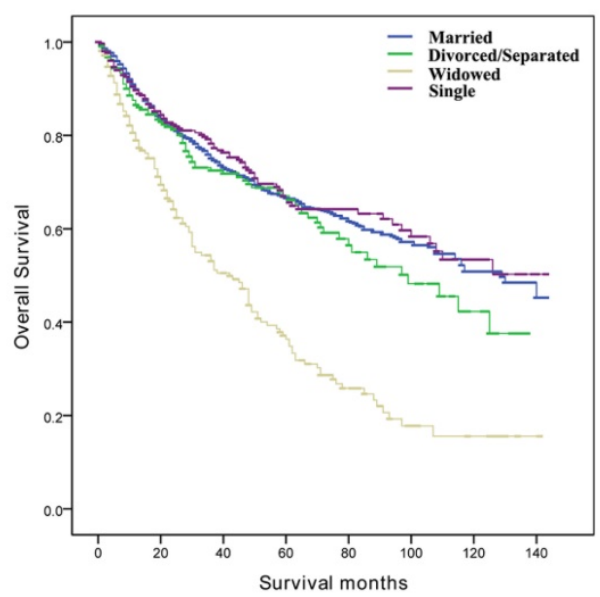

c.

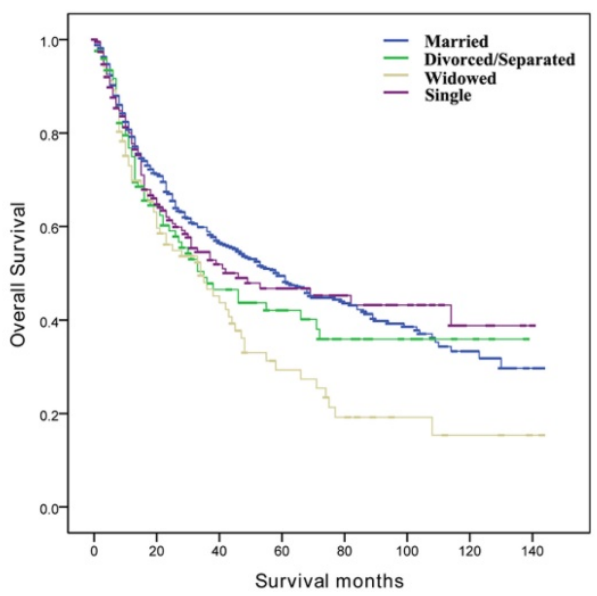

e.

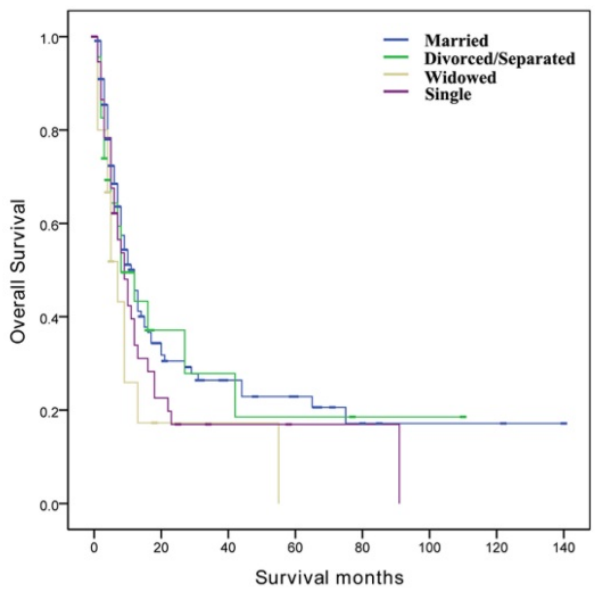

patients may not focus on their own health conditions, which makes it more likely that they will develop penile infections and increase the risk of illness [21].

\section{b.}

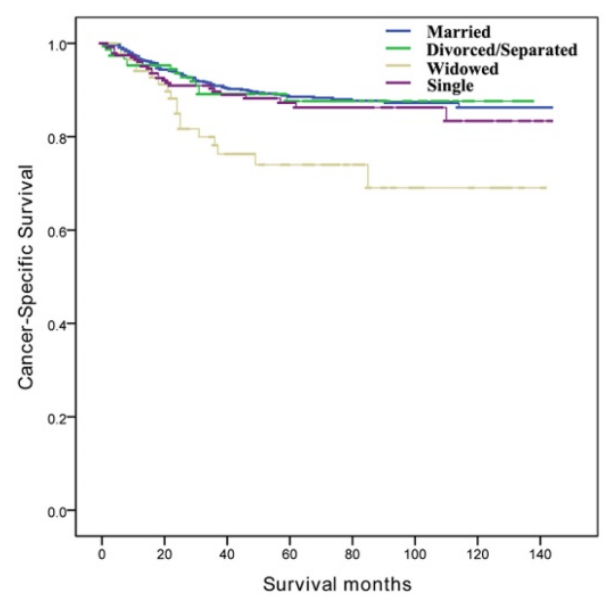

d.

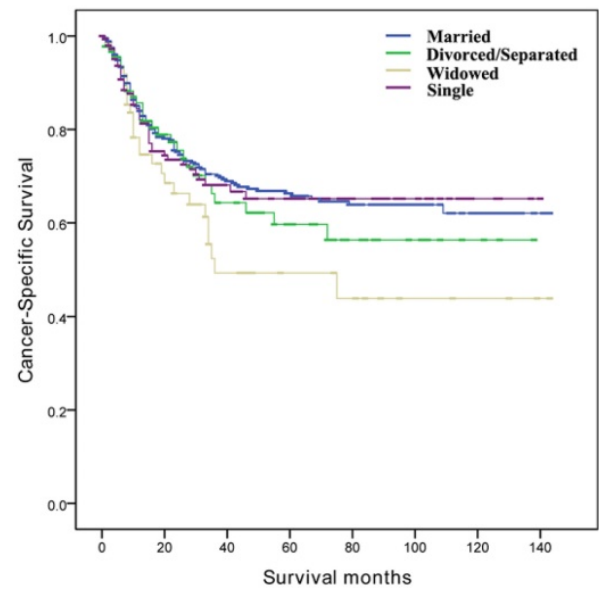

f.

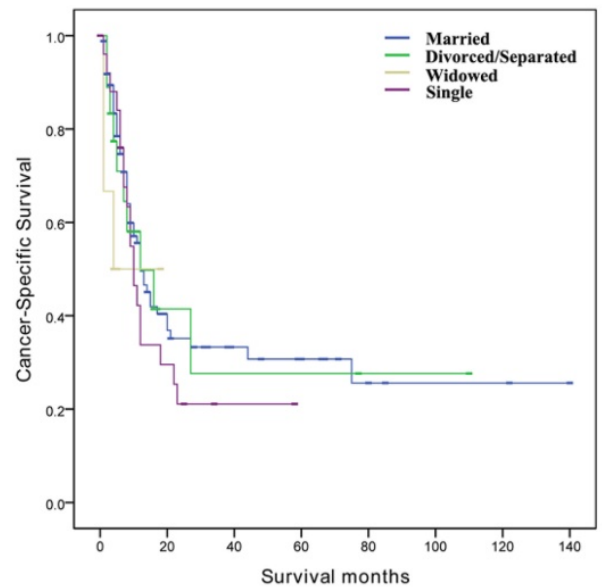

Figure 2. Overall survival and cancer-specific survival curves of penile cancer patients according to marital status in different SEER stages. a and $\mathbf{b}$, Overall survival and cancer-specific survival times in localized patients. $\mathbf{c}$ and $\mathbf{d}$, Overall survival and cancer-specific survival times in regional patients. e and $\mathbf{f}$, Overall survival and cancer-specific survival times in distant patients. 
a.

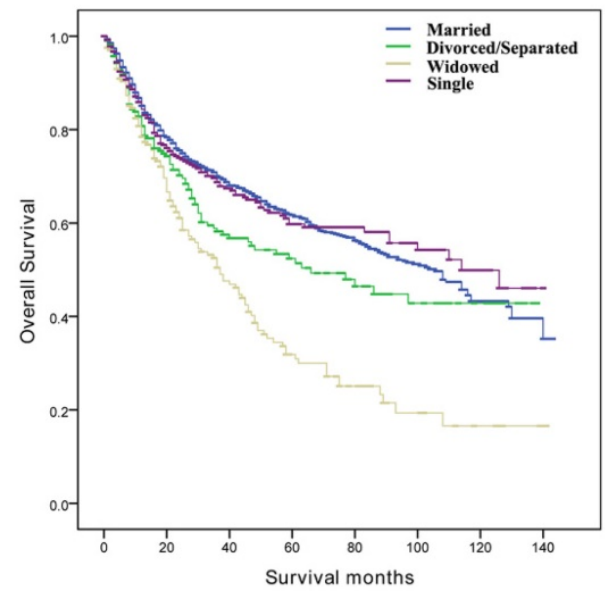

c.

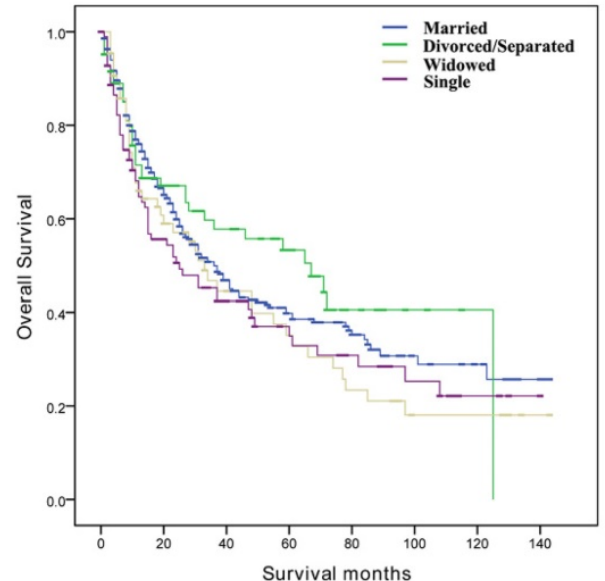

b.

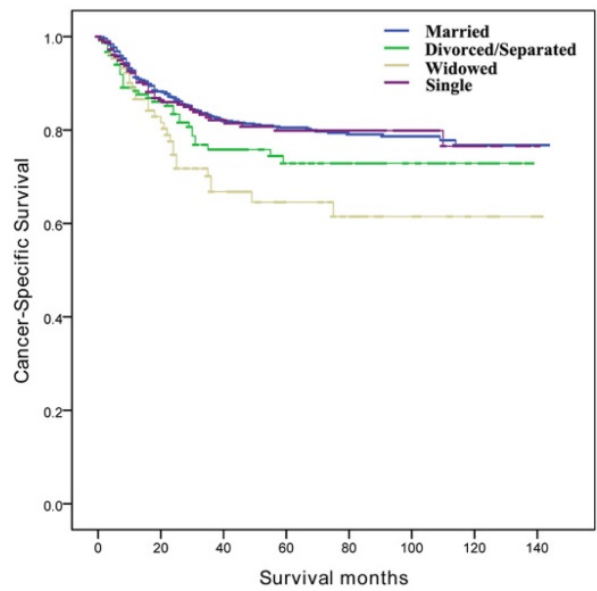

d.

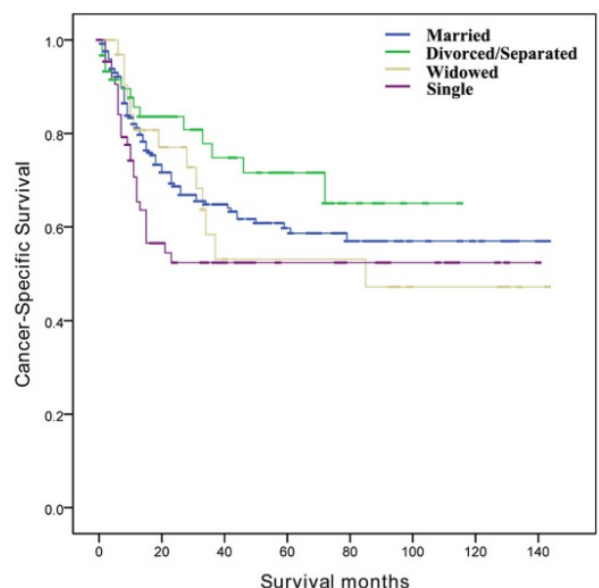

Figure 3. Overall survival and cancer-specific survival curves of penile cancer patients according to marital status in different grades. a and b, Overall survival and cancer-specific survival times in Grade I/Grade II patients. c and d, Overall survival and cancer-specific survival times in Grade III/Grade IV patients.

Previous studies have examined the relationship between marital status and the survival of patients with squamous cell carcinoma of the penis (SCCP). Thuret et al.[22] analysed 1,884 SCCP patients between 1988 and 2006 and found that unmarried men tend to present with less favourable disease stages when they have penile squamous cell carcinoma, and marital status had no effect on cancer-specific mortality. Ulff-Moller et al.[23] analysed the relationship between invasive penile squamous cell carcinoma and the marital statuses of 1,428 Danish patients and found that the divorced patients had the highest risk (HR: 1.49; 95\% CI: 1.24-1.79) compared with married men. However, our study focused on more types of penile cancer patients, including patients with penile squamous cell carcinoma and verrucous carcinoma. According to the marital status, we divided patients into four groups: married, divorced or separated, widowed, and single. In addition, we found that marital status was an independent prognostic factor for OS and CSS in patients with penile cancer. Moreover, the widowed patients showed the poorest OS (HR: 1.73; 95\% CI: 1.48-2.03, $p<0.001$ ) and the poorest CSS (HR: 1.64; 95\% CI: 1.144-1.279, $p<0.001)$ compared with married patients.

We analysed 69 patients who were diagnosed with penile cancer in our hospital between January 1 , 2013, and December 31, 2017, and found that 50 $(72.5 \%)$ patients were married, $1(1.5 \%)$ was divorced, $16(23.2 \%)$ were widowed, and $2(2.9 \%)$ were single. The Kaplan-Meier curves showed that the widowed patients have the worst survival times (Supplementary Figure S1). In addition, we found that, unlike the American population, our hospital had a higher proportion of widowed patients $(23.2 \%$ vs $10.2 \%$ ) but a lower proportion of divorced and single patients.

Furthermore, the lower survival times of widowed patients may be related to the following 
factors. First, widowed patients are more likely to be over 60 years old. Second, the immunity of the elderly population is low [24], thus resulting in the reduction of the OS of widowed patients. Finally, age affects the choice of treatment [25]. Older patients tend to choose more conservative treatments.

In addition, we found several interesting findings. For distant and Grade III/Grade IV subgroup patients, the effect of marital status on OS and CSS was not significant. This may be due to the higher degree of cancer risk in the distant and Grade III/Grade IV subgroup patients and to their poor physical conditions.

There were several limitations to our research. First, the SEER database is a retrospective dataset with its own retrospective study limitations. Second, the SEER database records the marital status of each patient at the time of diagnosis and lacks data on the subsequent changes in marital status. Third, the patient's physical condition was unclear, and patients with more comorbidities may pursue more conservative treatments. In addition, due to the small amount of data in our hospital, divorced and widowed patients were still alive at the end of the follow-up period. Therefore, further prospective clinical trials are necessary.

\section{Conclusions}

In our study, we found that marital status is an independent prognostic factor for survival in patients with penile cancer. Marital status has a protective effect on the OS and CSS of penile cancer. Unmarried penile cancer patients, especially widowed patients, are at greater risk for OS and CSS compared with married patients. Therefore, more health care should be provided for widowed patients.

\section{Supplementary Material}

Supplementary figure.

http://www.jcancer.org/v10p2661s1.pdf

\section{Acknowledgements}

The authors are grateful for the invaluable support and useful discussions with other members of the Urological Department.

\section{Funding}

This work was supported by grants from the National Natural Science Foundation of China (81001134) and Tongii University (1501219143) to Jiang Geng.

\section{Authors' Contributions}

WM, JF, JG were involved in the study conception and design. WM collected and assembled data. WM, JF and JG were involved in data analysis and interpretation $\mathrm{WM}, \mathrm{ZZ}$ wrote the manuscript.

\section{Competing Interests}

The authors have declared that no competing interest exists.

\section{References}

1. Takamoto D, Kawahara T, Kasuga J, Sasaki T, Yao M, Yumura Y, et al. The analysis of human papillomavirus DNA in penile cancer tissue by in situ hybridization. Oncology letters. 2018; 15: 8102-6.

2. Parkin DM, Bray F. Chapter 2: The burden of HPV-related cancers. Vaccine. 2006; 24 Suppl 3: S3/11-25.

3. Chaux A, Netto GJ, Rodriguez IM, Barreto JE, Oertell J, Ocampos S, et al. Epidemiologic profile, sexual history, pathologic features, and human papillomavirus status of 103 patients with penile carcinoma. World journal of urology. 2013; 31: 861-7.

4. Barski D, Georgas E, Gerullis H, Ecke T. Metastatic penile carcinoma - an update on the current diagnosis and treatment options. Central European journal of urology. 2014; 67: 126-32.

5. Backes DM, Kurman RJ, Pimenta JM, Smith JS. Systematic review of human papillomavirus prevalence in invasive penile cancer. Cancer causes \& control : CCC. 2009; 20: 449-57.

6. Schoen EJ, Oehrli M, Colby C, Machin G. The highly protective effect of newborn circumcision against invasive penile cancer. Pediatrics. 2000; 105: E36.

7. Wang L, Wilson SE, Stewart DB, Hollenbeak CS. Marital status and colon cancer outcomes in US Surveillance, Epidemiology and End Results registries: does marriage affect cancer survival by gender and stage? Cancer epidemiology. 2011; 35: 417-22.

8. Osborne C, Ostir GV, Du X, Peek MK, Goodwin JS. The influence of marital status on the stage at diagnosis, treatment, and survival of older women with breast cancer. Breast cancer research and treatment. 2005; 93: 41-7.

9. Shi RL, Chen Q, Yang Z, Pan G, Zhang Z, Wang W, et al. Marital status independently predicts gastric cancer survival after surgical resection--an analysis of the SEER database. Oncotarget. 2016; 7: 13228-35.

10. Wang XD, Qian JJ, Bai DS, Li ZN, Jiang GQ, Yao J. Marital status independently predicts pancreatic cancer survival in patients treated with surgical resection: an analysis of the SEER database. Oncotarget. 2016; 7: 24880-7.

11. Xu Z, Shi P, Yibulayin F, Feng L, Zhang H, Wushou A. Spindle cell melanoma: Incidence and survival, 1973-2017. Oncology letters. 2018; 16: 5091-9.

12. Gao Z, Ren F, Song H, Wang Y, Wang Y, Gao Z, et al. Marital Status and Survival of Patients with Chondrosarcoma: A Population-Based Analysis. Medical science monitor : international medical journal of experimental and clinical research. 2018; 24: 6638-48.

13. Xie JC, Yang S, Liu XY, Zhao YX. Effect of marital status on survival in glioblastoma multiforme by demographics, education, economic factors, and insurance status. Cancer medicine. 2018; 7: 3722-42.

14. Wu W, Fang D, Shi D, Bian X, Li L. Effects of marital status on survival of hepatocellular carcinoma by race/ethnicity and gender. Cancer management and research. 2018; 10: 23-32.

15. Aizer AA, Chen MH, McCarthy EP, Mendu ML, Koo S, Wilhite TJ, et al. Marital status and survival in patients with cancer. Journal of clinical oncology : official journal of the American Society of Clinical Oncology. 2013; 31: 3869-76.

16. Ayanian JZ, Kohler BA, Abe T, Epstein AM. The Relation Between Health-Insurance Coverage And Clinical Outcomes among Women with Breast-Cancer. New England Journal Of Medicine. 1993; 329: 326-31.

17. Kaiser NC, Hartoonian N, Owen JE. Toward a cancer-specific model of psychological distress: population data from the 2003-2005 National Health Interview Surveys. Journal of cancer survivorship : research and practice. 2010; 4: 291-302.

18. Satin JR, Linden W, Phillips MJ. Depression as a Predictor of Disease Progression and Mortality in Cancer Patients. Cancer. 2009; 115: 5349-61.

19. Goldzweig G, Andritsch E, Hubert A, Brenner B, Walach N, Perry S, et al Psychological distress among male patients and male spouses: what do oncologists need to know? Annals Of Oncology. 2010; 21: 877-83.

20. Ge LX, Mordiffi SZ. Factors Associated With Higher Caregiver Burden Among Family Caregivers of Elderly Cancer Patients A Systematic Review. Cancer nursing. 2017; 40: 471-8.

21. Kim A, Lee JA, Park HS. Health behaviors and illness according to marital status in middle-aged Koreans. J Public Health-Uk. 2018; 40: E99-E106.

22. Thuret R, Sun M, Budaus L, Abdollah F, Liberman D, Shariat SF, et al. A population-based analysis of the effect of marital status on overall and cancer-specific mortality in patients with squamous cell carcinoma of the penis. Cancer Cause Control. 2013; 24: 71-9.

23. Ulff-Moller CJ, Simonsen J, Frisch M. Marriage, cohabitation and incidence trends of invasive penile squamous cell carcinoma in Denmark 1978-2010. International journal of cancer. 2013; 133: 1173-9. 
24. Franceschi C, Capri M, Monti D, Giunta S, Olivieri F, Sevini F, et al. Inflammaging and anti-inflammaging: a systemic perspective on aging and longevity emerged from studies in humans. Mechanisms of ageing and development. 2007; 128: 92-105.

25. Serra-Rexach JA, Jimenez AB, Garcia-Alhambra MA, Pla R, Vidan M, Rodriguez P, et al. Differences in the therapeutic approach to colorectal cancer in young and elderly patients. The oncologist. 2012; 17: 1277-85. 